RESEARCH AND PRACTICE

\title{
The association between obesity, depression, and anxiety: Evidence from a community health needs assessment survey
}

\author{
Grace Bagwell Adams, PhD, MPA, and Angela Murcia \\ Department of Health Policy \& Management, University of Georgia, Athens, GA
}

\begin{abstract}
Background: The obesity epidemic has garnered much attention as a public health crisis. In order to make progress, it is necessary to understand the factors associated with obesity. One area of research needing more development is the link between obesity and other chronic health conditions. The objective of the present study was to further understand the relationship between obesity and the prevalence of anxiety and depression in Athens-Clarke County in northeast Georgia.
\end{abstract}

Methods: Data from the 2015 Athens-Clarke County Community Health Needs Assessment ( $n=1234)$ were used to assess the relationship between obesity and the prevalence of anxiety and depression among households at the county level. A linear probability model was utilized to estimate the effects of anxiety and depression, separately on the prevalence of household obesity. Household demographic and economic characteristics were applied as controls.

Results: The presence of anxiety in a household was associated with a $16 \%$ increase in the probability of obesity in the household $(\mathrm{p}<0.01)$ and the presence of depression was associated with a $21 \%$ increase in the probability that at least one household member was obese $(\mathrm{p}<0.01)$. Household demographics had no significant relationship with the presence of obesity, but neighborhood safety and receipt of Supplemental Nutrition Assistance Program (SNAP) were negatively associated with household obesity.

Conclusions: The results show a positive and statistically significant association between mental health and obesity. Although these findings are specific to Athens-Clarke County residents, the results are similar to those for national surveys, which find an association between obesity and mental health outcomes. Recommendations for public health organizations include the need for additional research and evaluation to create programs that address underlying issues pertaining to obesity.

Keywords: obesity, mental health, anxiety, depression

\section{INTRODUCTION}

In the United States, obesity has garnered attention as a primary public health challenge. In 2012, more than 33\% of adults and $17 \%$ of youth in the U.S. were obese (Ogden, Carroll, Kit, \& Flegal, 2014). Also in 2012, a greater percentage of the American populace was classified as overweight $-69 \%$ of adults and $24 \%$ of children (including those that were obese) (CDC National Center For Health Statistics, 2016). The implications of the obesity epidemic for the healthcare system are immense (Sturm, 2002). The cost of obesity accounted for $27 \%$ of the rise in healthcare spending between 1987 and 2001, a time of unprecedented growth in both obesity incidence and healthcare spending (Thorpe, Florence, Howard, \& Joski, 2004). In 2009, the annual medical cost of obesity to the US was $\$ 147$ billion; the cost was estimated to be $\$ 1,429$ higher for a person with obesity relative to an individual of normal weight (Finkelstein, Trogdon, Cohen, \& Dietz, 2009).

Increases in healthcare costs are linked to adverse health outcomes related to overweight and obesity in both children and adults. The motivations for the economic and public health concerns that obesity has been linked to causes of preventable death, including heart disease, stroke, type 2 diabetes, and certain types of cancer (Must et al., 1999; National Institute of Health, 1999). Research on the linkages between obesity and other chronic conditions is ongoing and is contributing to approaches for prevention and treatment of overweight and obesity.

The clinical and statistical significance of the link between obesity and mental health has been established (Gariepy, Nitka, \& Schmitz, 2010; Onyike, Crum, Lee, Lyketsos, \& Eaton, 2003). Anxiety and depression are two conditions found to be significantly associated with obesity. However, the reciprocal nature of the established relationship between obesity, anxiety, and depression makes it difficult to establish causality in one direction (Luppino et al., 2010). The complexity of obesity as a public health problem, especially as it relates to mental health, is increased by demographic disparities. Sex, race, ethnicity, education, and socioeconomic differences are each predictors of a person being obese (Gordon-Larsen, Nelson, Page, \& Popkin, 2006; Paeratakul, Lovejoy, Ryan, \& Bray, 2002; Wardle, Waller, \& Jarvis, 2002). Therefore, to address the problems posed by obesity, it is necessary to explore confounding factors such as socioeconomic status. 
The national obesity epidemic has considerable impact in the state of Georgia. Although slightly below the national average (30.5\%), the statewide prevalence of obesity has tripled in the last 25 years. Obesity is one a reason that many communities in the state of Georgia struggle to meet the economic and health needs of their residents. These communities provide an opportunity to evaluate the complex relationship between obesity and mental health.

Data from a recent Community Health Needs Assessment survey were used to assess the relationship between obesity and the prevalence of anxiety and depression among households in Athens-Clarke County (ACC), controlling for poverty. Athens Clarke County is a community with a significant percentage of low-income households. Since there is an empirical link between obesity and anxiety and depression, we hypothesized that households reporting the presence of anxiety and depression would be more likely to have obesity in the household. This county-level analysis should provide insight in regard to health disparities in the state of Georgia.

\section{METHODS}

Data were collected through a countywide Community Health Needs Assessment conducted in ACC from February through July of 2015. A population health survey instrument was created and administered to ACC households. The instrument was available in both English and Spanish. Participating households were notified about the Community Health Needs Assessment (CHNA) and the data collection methods online through social, professional, and neighborhood listservs and a website by means of which households could complete the survey instrument. Surveys were also administered in-person at community events hosted by public schools and local parks during the data collection period. To reach underrepresented populations, the surveys were also administered at monthly food pantries throughout the community.

The survey instrument included questions on demographic characteristics, socioeconomic status, health status, chronic conditions in the household (including obesity), prevalence of anxiety and depression, and additional questions on the neighborhood and built environment of responding households. The unit of analysis was the household-as respondents were asked to answer to survey questions for the household unit, rather than for individuals. The total sample size was 1,234 households.

Survey respondents were asked to identify household characteristics, including the number of people, their race and ethnicity, whether they participate in public programs such as SNAP, and health insurance status. In addition, respondents were asked if they felt that their neighborhood was safe. Three questions on health status of household members from the CHNA were used. These were: 1) Is anyone in your household (including you) obese? (Yes or No); 2) Does anyone in your household (including you) have anxiety? (Yes or No); and 3) Does anyone in your household (including you) have depression? (Yes or No).

Descriptive demographic information on the sample is provided in Table 1. Respondents indicated that $17.4 \%$ $(n=210)$ of households had at least one person who was obese at the time of the survey. Anxiety and depression were reported to be present in $23.0 \%(n=284)$, and $21.7 \%$ ( $n=268)$ of households, respectively. White households accounted for $65.0 \%$ of the sample $(n=802)$, African American households comprised $26.0 \%(n=321)$, and $4 \%$ $(n=49)$ of the sample identified as Latino. Only 1.2\% $(n=15)$ of households identified as Asian and 3.8\% $(n=47)$ as other (defined as Native American or Asian Pacific Islander). The average household was comprised of three persons.

Of the households, $11.0 \%(\mathrm{n}=136)$ indicated that they did not have health insurance (Table 1$)$. Only $65.8 \%(n=812)$ of survey participants indicated that they perceived their neighborhood as a safe place. Food assistance in the form of SNAP benefits (formerly known as Food Stamps) was received by $13.5 \%(n=167)$ of households; $3.2 \% \quad(n=39)$ indicated that the household received the Special Supplemental Nutrition for Women, Infants, and Children (commonly referred to as WIC).

Table 1. Descriptive Statistics of Community Health Needs Assessment Sample

\begin{tabular}{ll} 
& Mean \\
\hline Obesity in Household & $17.4 \%$ \\
Anxiety in Household & $23.0 \%$ \\
Depression in Household & $21.7 \%$ \\
White & $65.0 \%$ \\
African American & $26.0 \%$ \\
Latino & $4.0 \%$ \\
Asian & $1.2 \%$ \\
Other (Native American \& Pacific Islander) & $3.8 \%$ \\
Family Size & 2.97 \\
Uninsured & $11.0 \%$ \\
Neighborhood is Safe & $65.8 \%$ \\
SNAP & $13.5 \%$ \\
WIC & $3.2 \%$ \\
\hline Observations $(n)$ & 1234 \\
\hline
\end{tabular}


To examine the association between obesity and the mental health outcomes of anxiety and depression, a linear probability model was used. This approach allowed prediction of the probability that at least one person in the household was obese, as a function of household anxiety or depression. A linear probability model is appropriate when the dependent variable, or outcome of interest (in this case, obesity), is a dichotomous variable (Wooldridge, 2012). This means that the outcome-obesity-took on a value of 1 if the respondent indicated that at least one person in the household was obese and a value of 0 if the respondent said that no one in the household was obese.

The independent variables of interest were anxiety and depression, which were measured as dichotomous variables.
Because anxiety and depression were closely correlated, they were estimated in separate models using obesity as the dependent variable. Estimating anxiety and depression separately should yield more accurate results of the independent effect of each of these conditions on the presence of obesity. The linear probability model included control variables that were predictors of obesity status. These were the demographic and economic characteristics at the household level included in Table 1: race, ethnicity, family size, health insurance status, perception of neighborhood safety, SNAP participation, and WIC participation. All statistical analyses were conducted using STATA v.13.0 (Stata, 2013). Results of the two models are included in the table below.

Table 2. Relationship between Obesity and Mental Health Outcomes in Household

\begin{tabular}{|c|c|c|}
\hline & Obesity & Obesity \\
\hline Anxiety & $\begin{array}{c}0.16^{* * *} \\
(6.18)\end{array}$ & \\
\hline Depression & & $\begin{array}{c}0.21 * * * \\
(8.21)\end{array}$ \\
\hline White & $\begin{array}{l}-0.018 \\
(-0.34)\end{array}$ & $\begin{array}{l}-0.010 \\
(-0.20)\end{array}$ \\
\hline African American & $\begin{array}{l}0.0014 \\
(0.02)\end{array}$ & $\begin{array}{l}0.016 \\
(0.29)\end{array}$ \\
\hline Latino & $\begin{array}{l}-0.031 \\
(-0.41)\end{array}$ & $\begin{array}{l}-0.033 \\
(-0.44)\end{array}$ \\
\hline Asian & $\begin{array}{l}-0.093 \\
(-0.85)\end{array}$ & $\begin{array}{l}-0.084 \\
(-0.78)\end{array}$ \\
\hline Family Size & $\begin{array}{l}0.0087 \\
(1.56)\end{array}$ & $\begin{array}{c}0.0083 \\
(1.50)\end{array}$ \\
\hline Uninsured & $\begin{array}{l}0.027 \\
(0.76)\end{array}$ & $\begin{array}{l}0.022 \\
(0.64)\end{array}$ \\
\hline Neighborhood is Safe & $\begin{array}{c}-0.060 * * * \\
(-2.62)\end{array}$ & $\begin{array}{c}-0.057^{* *} \\
(-2.48)\end{array}$ \\
\hline SNAP is Received & $\begin{array}{c}-0.059 * \\
(-1.77)\end{array}$ & $\begin{array}{c}-0.078 * * \\
(-2.34)\end{array}$ \\
\hline WIC is Received & $\begin{array}{c}0.0088 \\
(0.14)\end{array}$ & $\begin{array}{l}0.031 \\
(0.48)\end{array}$ \\
\hline Constant & $\begin{array}{c}0.17^{* * *} \\
(3.02)\end{array}$ & $\begin{array}{c}0.15^{* * *} \\
(2.74)\end{array}$ \\
\hline Number of Observations & 1234 & 1234 \\
\hline
\end{tabular}

Table Notes: $* \mathrm{p}<0.10, * * \mathrm{p}<0.05, * * * \mathrm{p}<0.01$. Beta coefficients reported with t-statistics in parentheses. Logistic regressions were estimated with "obesity" as the dependent variable, measured as a " 0 " if no one is reported to be obese in the household and a " 1 " if obesity is present in the household. The first model reports results on the association between self-reported anxiety in the household and obesity, controlling for race, family size, insurance status, neighborhood safety, and receipt of SNAP and WIC. The second model reports results on the association between self-reported depression in the household and obesity, controlling for race, family size, insurance status, neighborhood safety, and receipt of SNAP and WIC. Reference category for race is "other," defined as Native American or Asian Pacific Islander. 


\section{RESULTS}

Table 2 presents results of the anxiety and depression models. Results from the first linear probability model show a statistically significant relationship between anxiety and prevalence of obesity in the household. The presence of anxiety is associated with a $16 \%$ increase in the probability of obesity in the household $(\mathrm{p}<0.01)$. Conversely, there was no significant relationship between obesity and any of the demographic controls in the model (race, ethnicity, or family size).

Additional relationships of significance include the perception of neighborhood safety and receipt of SNAP benefits. Respondents who perceived that their household was in a safe neighborhood showed a negative association with prevalence of obesity in the household. Neighborhood safety was associated with a $6 \%$ decrease in the probability that at least one person in the household is obese $(p<0.01)$. Receipt of SNAP was negatively associated with obesity; SNAP receipt is associated with a $5.9 \%$ decrease in the probability of obesity in the household $(\mathrm{p}<0.10)$.

The second set of results (Table 2) are findings on the association between depression and obesity. As in the anxiety model, there was a statistically significant relationship between depression and obesity. The presence of depression in a household was associated with a $21 \%$ increase in the probability that at least one household member was obese $(\mathrm{p}<0.01)$. Race, ethnicity, and family size had no significant relationship with the presence of obesity.

The perception of neighborhood safety was associated with a $5.7 \%$ decrease in the probability that obesity was present in the household $((\mathrm{p}<0.05)$. Among those households receiving SNAP, there was a $7.8 \%$ decrease in the probability of obesity in the household $(p<0.05)$. This finding is similar to that for the anxiety model, but the magnitude and significance of the negative association between SNAP receipt and obesity is larger in the second model. As a sensitivity analysis, stepwise regressions were conducted for both the anxiety and depression models; the results showed findings similar to those discussed above; anxiety and depression were significant in each model, as were neighborhood safety and receipt of SNAP.

\section{DISCUSSION}

The results show a statistically significant association between mental health and obesity. Households with selfreported anxiety are associated with a $16 \%$ increase in the likelihood of having at least one obese person. The association with depression is greater-households with self-reported depression are associated with a $21 \%$ increase in the probability of obesity. These findings are specific to ACC residents but are similar to larger, national surveys that find an association between obesity and mental health outcomes (Luppino et al., 2010; Onyike et al., 2003). The directional nature of the relationship was not determined.
Stress could be a variable influencing both mental health and obesity. The relationships between stress and depression and between stress and anxiety have been established (Finlay-Jones \& Brown, 1981; Hammen, 2005). There are also links between stress, anxiety and depression, and obesity (Finlay-Jones \& Brown, 1981; Hammen, 2005; Luppino et al., 2010) . A relevant pathways involves the influence of stress on eating habits, where chronic stress leads to a preference for foods high in fat and sugar (Torres \& Nowson, 2007). Stress-induced eating could be a causal link between mental health and obesity.

In this model, two other findings were consistent. First, neighborhood safety is negatively and significantly associated with incidence of obesity in the household. The built environment has substantial impact on the public health of communities. There is evidence of a link between socioeconomic disparities and access to outdoor facilities (Gordon-Larsen et al., 2006). When families feel safe in their neighborhood, they may be more likely to go outside and engage in physical activity. This could include walking or running in the neighborhood or playing in their yards. Safe neighborhoods might also have better access to parks or other recreational facilities, such as playgrounds. Conversely, if the neighborhood is perceived as unsafe, family members may be less likely to engage in physical activity. The link between neighborhood safety and prevalence of obesity should be considered as public health interventions are developed and implemented.

Of interest also is the negative and significant association between receipt of SNAP and obesity. Individuals receiving SNAP are eligible through meeting certain requirements, including family size and income. Recipients must fall at or below $130 \%$ of the Federal Poverty Line. These households generally have budget constraints and may struggle to have enough resources to buy adequate food for all household members. Thus, there is an association between low-income households receiving food assistance and the prevalence of household obesity. Poverty, which is linked to food insecurity (defined as inadequate access to enough food to live a healthy life), could be driving the negative relationship between receipt of SNAP and obesity.

This study has several limitations. First, the sampling methodology did not yield a representative sample. The way in which survey respondents were recruited included attempts to achieve a diverse sample (e.g., low-income participants were surveyed in-person at the local food bank), but this convenience sample may or may not be representative of the entire population of ACC. Second, the self-reporting nature of the survey might bias the results towards conservative estimates of the prevalence of chronic conditions or mental health issues, especially considering the stigma that might lead respondents to underreport conditions such as anxiety or depression. Self-reporting of obesity could also bias the measured prevalence of obesity. Respondents did not report their height and weight; they reported only whether anyone in their household was obese. Third, respondents answered survey questions for the household rather than as individuals, which could also bias the results. These shortcomings limit our ability to obtain 
true estimates of the prevalence of these conditions and the relationship between mental health and obesity. Fourth, this study is limited by the cross-sectional nature of the datathe CHNA survey was a snapshot of ACC households at one point in time. We are unable to establish causality and report only associations between variables of interest because we did not observe respondents over time. Last, because of the local nature of this study, we cannot assume that the findings are generalizable to other communities in the state of Georgia.

\section{CONCLUSIONS}

While there have been advances in research focusing on understanding the relationship between obesity and chronic conditions, there are gaps that need to be explored. Particularly, knowledge of this relationship at the local level is limited. The present study used county-level data to examine the relationship between mental health (specifically, anxiety and depression) and obesity.

Despite the fact that the findings are specific to residents of one county, the results are very similar to those of larger research studies that have found associations between obesity and mental health outcomes. The link between these health areas lead to the following recommendation: (a) Public health organizations should engage in additional research that includes measures of mental health to address issues pertaining to obesity. (b) To improve the efficacy of obesity-targeted public health programs, evidence-based interventions should integrate information on the links between stress, mental health, and obesity.

The association observed between obesity and mental health conditions such as anxiety and depression points to an area of improvement in the development of public health interventions targeting obesity. A practical application is the integration of physical and mental health screening tools in initial and follow-up health assessments. This change could allow public health officials to target health resources towards persons at a higher risk for mental health conditions and obesity.

Another implication for public health is the prospect of using mental health interventions as a gateway for nutrition education, opportunities and incentives for physical activity, and physical health support, all of which could decrease the incidence and prevalence of obesity among the segment of the population with mental health conditions. Leveraging these findings on the link between mental health and obesity could improve the effectiveness of public health interventions in communities across the state and reduce the incidence of obesity.

\section{Acknowledgements}

The authors would like to acknowledge Community Connection of Northeast Georgia, Fenwick Broyard, and Emily Hui for conducting the Community Health Needs Assessment and data collection efforts, and the Athens Regional Health System and St. Mary's Healthcare System of Athens for supporting the 2015 Athens Clarke County Community Health Needs Assessment.

\section{References}

CDC National Center For Health Statistics. (2015, September 30, 2015 ). Obesity and Overweight.

Finkelstein, E., Trogdon, J., Cohen, J., \& Dietz, W. (2009). Annual medical spending attributable to obesity: payer-and servicespecific estimates. Health affairs, 28(5), 822-831.

Finlay-Jones, R., \& Brown, G. W. (1981). Types of stressful life event and the onset of anxiety and depressive disorders. Psychological Medicine, 11(04), 803-815.

Gariepy, G., Nitka, D., \& Schmitz, N. (2010). The Association Between Obesity and Anxiety Disorders in the Population: A Systematic Review and Meta-analysis. International journal of obesity, 34(3), 407-419.

Gordon-Larsen, P., Nelson, M. C., Page, P., \& Popkin, B. M. (2006). Inequality in the built environment underlies key health disparities in physical activity and obesity. Pediatrics, 117(2), 417-424.

Hammen, C. (2005). Stress and depression. Annu. Rev. Clin. Psychol., 1, 293-319.

Luppino, F., de Wit, L., Bouvy, P., Stijnen, T., Cuijpers, P., Penninx, B., \& Zitman, F. (2010). Overweight, Obesity, and Depression: A Systematic Review and Meta-analysis of Longitudinal Studies. Archives of general psychiatry, 67(3), 220-229.

Must, A., Spadano, J., Coakley, E. H., Field, A. E., Colditz, G., \& Dietz, W. H. (1999). The disease burden associated with overweight and obesity. Jama, 282(16), 1523-1529.

National Institute of Health. (1998). Clinical Guidelines on the Identification, Evaluation, and Treatment of Overweight and Obesity in Adults. Retrieved from

Ogden, C., Carroll, M., Kit, B., \& Flegal, K. (2014). Prevalence of Childhood and Adult Obesity in the United States, 2011-2012. Jama, 311(8), 806-814.

Onyike, C. U., Crum, R. M., Lee, H. B., Lyketsos, C. G., \& Eaton, W. W. (2003). Is obesity associated with major depression? Results from the Third National Health and Nutrition Examination Survey. American journal of epidemiology, 158(12), 1139-1147.

Paeratakul, S., Lovejoy, J., Ryan, D., \& Bray, G. (2002). The relation of gender, race and socioeconomic status to obesity and obesity comorbidities in a sample of US adults. International journal of obesity and related metabolic disorders: journal of the International Association for the Study of Obesity, 26(9), 1205-1210.

Stata. (2013). Release 13: Statistical software College Station, TX: StataCorp LP.

Sturm, R. (2002). The effects of obesity, smoking, and drinking on medical problems and costs. Health affairs, 21(2), 245-253.

Thorpe, K., Florence, C., Howard, D., \& Joski, P. (2004). The Impact of Obesity on Rising Medical Spending. Health affairs, 23, 283-283.

Torres, S. J., \& Nowson, C. A. (2007). Relationship between stress, eating behavior, and obesity. Nutrition, 23(11), 887-894.

Wardle, J., Waller, J., \& Jarvis, M. J. (2002). Sex differences in the association of socioeconomic status with obesity. American Journal of Public Health, 92(8), 1299-1304.

Wooldridge, J. (2012). Introductory econometrics: A modern approach: Cengage Learning. 